\title{
Genetic diversity and pathogenicity of Fusarium species associated with fruit rot disease in banana across Peninsular Malaysia
}

\begin{abstract}
Aims: The aims of this study were to identify the Fusarium isolates based on translation elongation factor (tef) $1 \alpha$ sequence, to determine the genetic diversity among isolates and species using selected microsatellite markers and to examine the pathogenicity of Fusarium isolates causing fruit rot disease of banana. Methods and Results: One-hundred and thirteen microfungi isolates were obtained from fruit rot infected banana in Peninsular Malaysia. However, this study was focused on the dominant number of the discovered microfungi that belongs to the genus Fusarium; 48 isolates of the microfungi have been identified belonging to 11 species of Fusarium, namely Fusarium incarnatum, Fusarium equiseti, Fusarium camptoceras, Fusarium solani, Fusarium concolor, Fusarium oxysporum, Fusarium proliferatum, Fusarium verticillioides, Fusarium sacchari, Fusarium concentricum and Fusarium fujikuroi. All Fusarium isolates were grouped into their respective clades indicating their similarities and differences in genetic diversity among isolates. Out of 48 Fusarium isolates tested, 42 isolates caused the fruit rot symptom at different levels of severity based on Disease Severity Index (DSI). The most virulent isolate was F. proliferatum B2433B with DSI of 100\%. Conclusions: All the isolated Fusarium species were successfully identified and some of them were confirmed as the causal agents of pre- and postharvest fruit rot in banana across Peninsular Malaysia. Significance and Impact of the Study: Our results will provide additional information regarding new report of Fusarium species in causing banana fruit rot and in the search of potential biocontrol agent of the disease.
\end{abstract}

Keyword: Disease; Diversity; Genetic; Identification; Pathogenesis 